\title{
MICROBIOLOGICAL SURVEILLANCE OF INTENSIVE CARE UNITS IN A TERTIARY CARE TEACHING HOSPITAL-WESTERN INDIA
}

\author{
RAVAL P.N. ${ }^{1}$, PATEL P.G. ${ }^{1}$, PATEL B.V. ${ }^{1}$, SONI S.T. ${ }^{*}$, BHATT S.K. ${ }^{1}$, VEGAD M.M. ${ }^{1}$, VAGHELA G.M. ${ }^{2}$, \\ YADAV K.S. ${ }^{3}$, SHIRISH PATIL ${ }^{3}$ AND GOSWAMI H.M. ${ }^{1}$
}

1B.J. Medical College, Civil Hospital, Ahmedabad- 380016, Gujarat, India.

2Government Medical College, Surat-395001, Gujarat, India.

3Padmashree Dr. D.Y. Patil Medical College and Hospital, Navi Mumbai- 400706, MS, India.

*Corresponding Author: Email-drsumeetasoni@gmail.com

Received: July 25, 2012; Accepted: August 02, 2012

\begin{abstract}
-
Introduction- Infection is a commonly encountered problem for patients in intensive care units (ICUs) and Multidrug-resistant bacterial infection is predominant. In intensive care unit (ICU) isolation, identification and antibiotic susceptibility pattern of microbiological isolate is important because of increased rate of mortality and morbidity and their economic burden which constitutes serious problem around the globe.
\end{abstract}

Materials and Methods- This study was conducted over a period of one year in the different ICUs of a tertiary care, teaching Hospital in Western India. Different clinical samples were collected and analyzed by routine conventional methods in a Microbiology laboratory. Antibiotic susceptibility (ABS) test was done using modified Kirby-Bauer disk diffusion method as per CLSI guidelines.

Results- Total 1145 clinical samples were processed, out of which 554 showed evidence of infection, 469 were gram-negative, 64 were gram-positive and 21 were fungal infection specially candida species. Urinary tract infections and Skin and soft tissue infections were most common infection in MICU/PICU and SICU respectively. Among the isolates, the most commonly found microorganism was Pseudomonas spp. in skin lesions, soft tissue wounds \& in blood. However $E$. coli was predominant in urinary tract infection and Klebsiella pneumoniae in respiratory tract infection. The majority of the Gram-negative isolates were resistant to multiple antibiotics while $59.46 \%$ of Staphylococcus aureus isolates were Methicillin Resistant Staphylococcus aureus (MRSA).

Conclusion- Outcomes of this study is highlighting the significance of microbial isolation \& identification in patients in Intensive care Units (ICU) and shall improve antibiotic choices for number of patients admitted each year. Regular surveillance of antibiotic susceptibility pattern is important clue to clinicians in choosing antibiotics for the patients in ICU.

Key Words- microbiological isolates, antibiotic susceptibility, multidrug resistant organisms, intensive care unit

Citation: Raval P.N., et al (2012) Microbiological Surveillance of Intensive Care Units in a Tertiary Care Teaching Hospital-Western India. International Journal of Microbiology Research, ISSN: 0975-5276 \& E-ISSN: 0975-9174, Volume 4, Issue 7, pp.-270-274.

Copyright: Copyright@2012 Raval P.N., et al. This is an open-access article distributed under the terms of the Creative Commons Attribution License, which permits unrestricted use, distribution and reproduction in any medium, provided the original author and source are credited.

\section{Introduction}

Infection remains a major problem for patients in intensive care units (ICUs) and is associated with considerable morbidity, mortality, and direct cost to the patient and indirect to the hospital by way of hospital acquired infection. Infection and related sepsis are the leading cause of death in non-cardiac ICUs where the mortality rates is around $60 \%$ and it contribute for approximately $40 \%$ of total ICU expenditures. Importantly, the incidence of sepsis is increasing the number of infection- related deaths [1].
Antimicrobial resistance in ICU infections is increasing worldwide. Both morbidity and mortality is greater when infection is caused by drug resistant organisms. This is due to overuse and misuse of antimicrobial agents, immunocompromised conditions of patients and exogenous transmission of bacteria, usually by hospital personnel [2]. Knowledge of an ICU's most common bacterial isolates and their antibiotic susceptibility patterns facilitates effective empirical antibiotic therapy and supports decisions to restrict or reduce the clinical availability of certain antibiotics. Antibiotic interventions 
should aim to limit the emergence of antibiotic resistance whilst simultaneously improving patient outcomes and decreasing drug costs [3].

The important step in the prevention and control of infection in the ICU is effective hospital infection control policy, including hand washing and specific surveillance for the most prevalent infections. Educating healthcare professionals and heightening their awareness of these objectives is the key to success [4]. Because of importance of ICU infections, surveillance studies are important to obtain the necessary data regarding the regional microbiological isolates and their susceptibility to antibiotics. Therefore, this study was conducted to determine the spectrum and their antibiotic resistance in different ICUs in Tertiary care Teaching Hospital, Western India.

\section{Materials and Methods}

The present study is conducted at Civil Hospital, Ahmedabad. It is a tertiary care and teaching hospital treating about 6 to 6.5 lakh outdoor patients, admitting 70,000 patients annually. Over the period of one year between June 2010 and June 2011, various samples from patients admitted in different intensive care units in hospital were collected for culture and sensitivity. Core specialty, medical history, gender and microbial isolates was recorded for all patients whose samples were collected for analysis. This study comprised of 1145 clinical specimens like blood, urine, pus, post surgical wounds swabs, sputum and body fluid from patients of all age group admitted in ICU.

Gram staining were done on the primary smears. Samples were inoculated on Nutrient agar, blood agar and MacConkey's agar. The blood agar plates were incubated in presence of $5 \% \mathrm{CO}_{2}$ at $37{ }^{\circ} \mathrm{C}$ and both MacConkey's agar and nutrient agar plates were incubated aerobically at $37^{\circ} \mathrm{C}$ for 24 hours. After overnight incubation of the plates bacterial growth were isolated and identified on the basis of gram stain from culture growth, \& biochemical characteristics, as per standard CLSI guidelines [5]. All the organisms isolated were tested for antibiotic susceptibility pattern on MuellerHinton Agar media using modified Kirby-Bauer disk diffusion method as per Clinical and Laboratory Standards Institute (CLSI) guideline [5].

Quality Control-Standard strains Staphylococcus aureus ATCC 25923; Escherichia coli ATCC 25922 and Pseudomonas aeruginosa ATCC 27853 were used. The diameter of the zone of inhibition of growth was recorded and interpreted as susceptible, intermediate or resistant. Screening and confirmation of Methicillin Resistant Staphylococcus aureus (MRSA), Extended spectrum $\beta$ lactamase (ESBL), Amp C, Carbapenamase and Metallo $\beta$ lactamase (MBL) producing isolates were done separately.

\section{Results}

\section{Study Population}

During the study period, total 1145 samples were received from the patients admitted in Intensive care unit in Civil Hospital, Ahmedabad. From the total 1145 specimens obtained, 718 (62.71\%) were from male patients and 427 (37.29\%) from females. Mean age for patients admitted in ICU was 41.88 years. Out of total 1145 samples received, 554 (48.38\%) specimens were culture positive and
$591(51.62 \%)$ specimens showed no growth. Out of total 554 positive isolates, $341(61.55 \%)$ and $213(38.45 \%)$ were from Surgical and Medical/Pediatric ICU respectively. Infection rate was higher in surgical ICUs (56.18\%) than in medical/pediatric ICUs (39.59\%).

Table 1- Gender and core specialty distribution of Patients admitted in the ICU

\begin{tabular}{|lllll|}
\hline Characteristic & \multicolumn{2}{c}{ Total Patients Infected } & \multicolumn{2}{l}{ Not Infected Infection Rate } \\
Age in years (mean) & 41.88 & 43.55 & 40.32 & -- \\
Male & $718(62.71 \%)$ & 385 & 333 & $53.62 \%$ \\
Female & $427(37.29 \%)$ & 169 & 258 & $39.58 \%$ \\
Total patients & $1145(100 \%)$ & 554 & 591 & -- \\
Medical ICU & $538(46.99 \%)$ & 213 & 325 & $39.59 \%$ \\
Surgical ICU & $607(53.01 \%)$ & 341 & 266 & $56.18 \%$ \\
Total patients & $1145(100 \%)$ & 554 & 591 & -- \\
\hline
\end{tabular}

Table 2 represents the age wise distribution of patients in this study. Infection rate was highest in $0-9$ years age group (65.22\%) followed by $>60$ years (57.22\%), 40-49 years (51.87\%), 50-59 years $(51.39 \%)$. Infection rate in age group between 10 to 39 years range from $35 \%$ to $43 \%$.

Table 2- Pediatric and non-pediatric distribution of infected and non-infected patients

\begin{tabular}{|llll|}
\hline Age & Total Patients & \multicolumn{2}{l}{ Infected Patients Infection rate \% } \\
\hline $0-9$ & 23 & 15 & 65.22 \\
$10-19$ & 89 & 38 & 42.7 \\
$20-29$ & 136 & 48 & 35.29 \\
$30-39$ & 215 & 91 & 42.33 \\
$40-49$ & 214 & 111 & 51.87 \\
$50-59$ & 288 & 148 & 51.39 \\
$>60$ & 180 & 103 & 57.22 \\
Total number of patients & 1145 & 554 & -- \\
\hline
\end{tabular}

\section{Prevalence and Characteristics of Infections}

During June, 2010 to June, 2011, total 1145 different clinical specimens like blood, urine, pus, post surgical wounds swabs, sputum and body fluid were collected from patients admitted in Intensive care unit. Out of them, 554 (48.38\%) specimens were culture positive and 591 (51.62\%) specimens showed no growth. Total 469 $(84.65 \%)$ isolates were gram negative, 64 (11.55\%) were gram positive and $21(3.79 \%)$ were candida spp. Maximum numbers of pathogens were isolated from skin lesions, soft tissue wounds, urine, blood and sputum.

In surgical ICUs, Skin and soft tissue infections (63.64\%) were most predominant infections, followed by urinary tract infections $(16.13 \%)$, Blood stream infections $(8.80 \%)$ and Respiratory tract infections $(5.87 \%)$. While in Medical/Pediatric ICUs, urinary tract infections $(30.99 \%)$ were most predominant infections, followed by Skin and soft tissue infections (23.47\%), Blood stream infections (17.37\%) and Respiratory tract infections (16.90\%).

Table 3- Site wise distribution of positive isolates in Medical/ Pediatric and Surgical ICUs

\begin{tabular}{lllll|} 
Site & Medical/ Paediatric ICU & $\%$ & Surgical ICU & $\%$ \\
\hline Skin and Soft tissue & 50 & 23.47 & 217 & 63.64 \\
Urinary tract & 66 & 30.99 & 55 & 16.13 \\
Blood stream & 37 & 17.37 & 30 & 8.8 \\
Respiratory tract & 36 & 16.9 & 20 & 5.87 \\
Other sites & 24 & 11.27 & 19 & 5.57 \\
Total & 213 & 100 & 341 & 100 \\
\hline
\end{tabular}




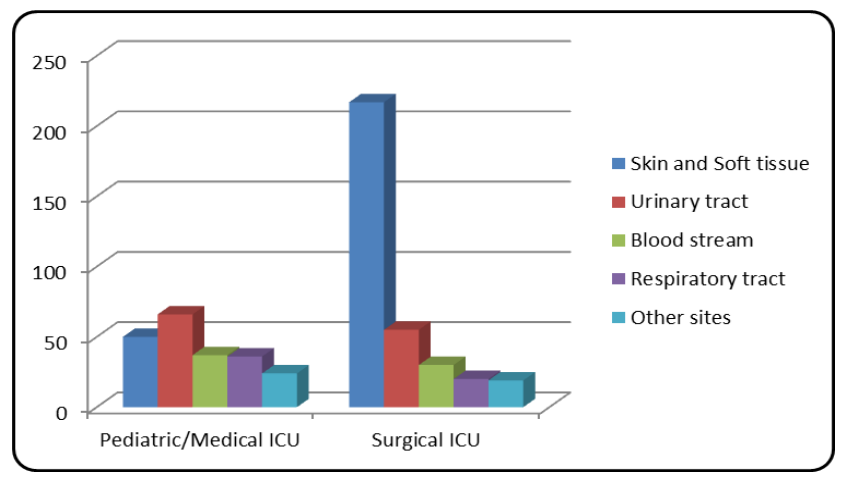

Fig. 1- Source of infection in Medical/ Paediatric ICU \& in Surgical ICU

Pseudomonas spp. was found to be most predominant isolate (27.62\%) found from ICU patients followed by Klebsiella pneumoniae $(20.76 \%)$, E. coli (19.68\%), Acinetobacter spp. $(14.62 \%)$ and Staphylococcus aureus (6.68\%). According to clinical specimens, the most commonly isolated microorganism was Pseudomonas spp. in skin, soft tissue \& blood stream infection, E. coli in urinary tract infection and Klebsiella pneumoniae in respiratory tract infection.

\begin{tabular}{|c|c|c|c|c|c|c|}
\hline \multirow[b]{2}{*}{ Organisms } & \multicolumn{5}{|c|}{ Site of infection } & \multirow[b]{2}{*}{ Total } \\
\hline & $\begin{array}{l}\text { Skin and } \\
\text { Soft tissue }\end{array}$ & $\begin{array}{l}\text { Urinary } \\
\text { tract }\end{array}$ & $\begin{array}{l}\text { Blood } \\
\text { stream }\end{array}$ & $\begin{array}{l}\text { Respiratory } \\
\text { tract }\end{array}$ & $\begin{array}{l}\text { Other } \\
\text { sites }\end{array}$ & \\
\hline Pseudomonas spp. & 84 & 26 & 21 & 13 & 9 & 153 \\
\hline Klebsiella pneumoniae & 64 & 12 & 6 & 19 & 14 & 115 \\
\hline E. coli & 42 & 55 & 3 & 3 & 6 & 109 \\
\hline Acinetobacter spp. & 45 & 2 & 17 & 14 & 3 & 81 \\
\hline S. aureus & 11 & 0 & 17 & 2 & 7 & 37 \\
\hline Candida spp. & 2 & 12 & 1 & 4 & 2 & 21 \\
\hline Other Organisms & 19 & 14 & 2 & 1 & 2 & 38 \\
\hline Total & 267 & 121 & 67 & 56 & 43 & 554 \\
\hline
\end{tabular}

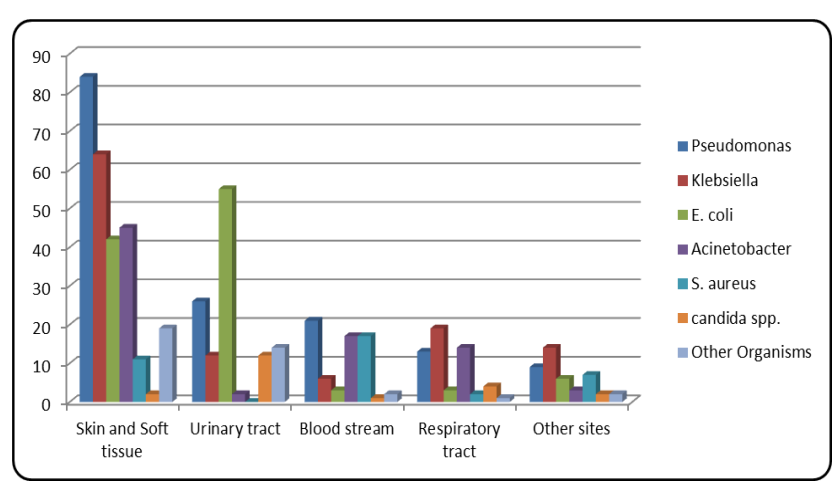

Fig. 2- Specimen wise incidence of organisms isolated form clinical samples

Most common species identified among Pseudomonas isolates was Pseudomonas aeruginosa (140/153) followed by Burkholderia cepacia complex (7/153), Stenotrophomonas maltophilia (4/153), Pseudomonas putida (2/153). In case of Acinetobacter spp, most common species were $A$. baumannii (75/81) followed by A. Iwoffi (4/81) and A. junii (2/81). Among 21 Candida spp, 11 were Candida albicans and 10 were Candida non albicans. Among non albicans Candida tropicalis (8/10) was the most frequent isolate fol- lowed by Candida glabrata (2/10).

Antibiotic resistance patterns revealed that majority of bacterial isolates were resistant to multiple antibiotics. Table 5 \& Fig. 3 explains the resistance pattern of different microorganisms to common antibiotics.

Table 5- Antibiotic Resistance patterns of various Organisms isolated from patients admitted in MICU, PICU and SICU of tertiary care centre.

\begin{tabular}{|c|c|c|c|c|}
\hline \multirow{3}{*}{$\begin{array}{l}\text { Antibiotic } \\
\text { Cefaclor } \\
\text { Cefoperazone }\end{array}$} & \multicolumn{4}{|c|}{ Resistance Rate in Different Organisms (\%) } \\
\hline & -- & 97.35 & 95.06 & - \\
\hline & 68.63 & -- & -- & -- \\
\hline Ceftazidime & 72.55 & -- & -- & -- \\
\hline Cefotaxime & -- & 95.59 & 87.65 & -- \\
\hline Cefepime & -- & 82.37 & 76.54 & \\
\hline $\begin{array}{l}\text { Piperacillin- } \\
\text { tazobactam }\end{array}$ & 16.99 & 49.33 & 20.98 & -- \\
\hline $\begin{array}{l}\text { Ampicillin- } \\
\text { sulbactam }\end{array}$ & -- & 52.42 & 19.75 & -- \\
\hline Imipenem & 10.46 & 3.08 & 7.4 & -- \\
\hline Gentamycin & -- & 77.97 & 77.77 & 56.75 \\
\hline Tobramycin & 80.39 & 77.97 & -- & -- \\
\hline Clindamycin & -- & -- & -- & 48.65 \\
\hline Ciprofloxacin & 79.08 & 71.37 & -- & -- \\
\hline Levofloxacin & 23.53 & 14.01 & 17.28 & 21.62 \\
\hline Aztreonam & 30.07 & & -- & -- \\
\hline Amikacin & 35.94 & 41.4 & 53.09 & -- \\
\hline Azithromycin & -- & & -- & 83.78 \\
\hline Tetracycline & -- & 78.41 & 75.31 & 56.76 \\
\hline Cotrimoxazole & -- & 81.93 & 83.95 & 45.95 \\
\hline $\begin{array}{l}\text { Chloramphen- } \\
\text { icol }\end{array}$ & - & -- & -- & 35.14 \\
\hline Linezolid & -- & -- & -- & 0 \\
\hline Cefoxitin & -- & -- & -- & 59.46 \\
\hline Vancomycin & -- & -- & -- & 0 \\
\hline Polymyxin B & 4.58 & 9.69 & 2.47 & -- \\
\hline Tigecycline & -- & 7.05 & 9.88 & 0 \\
\hline
\end{tabular}

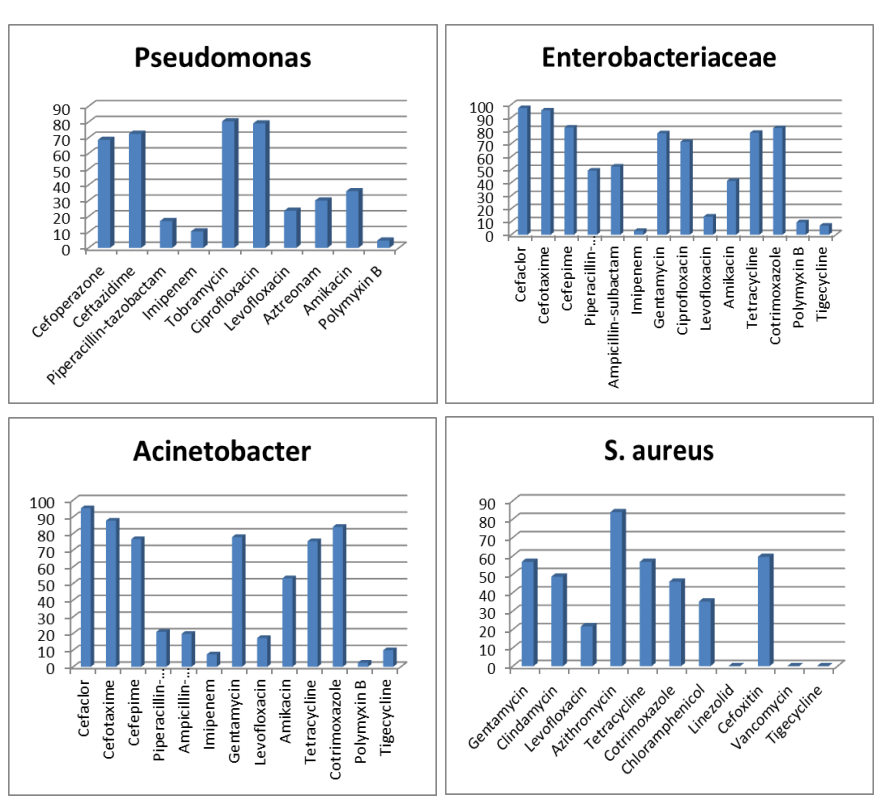

Fig. 3- Antibiotic resistance pattern of different isolates

Pseudomonas spp. showed least rate of resistance to Polymyxin B (4.58\%), imipenem (10.46\%) and piperacillin-tazobactam (16.99\%). 
They showed moderate degree of resistance to levofloxacin $(23.53 \%)$, aztreonam $(30.07 \%)$ and amikacin $(35.94 \%)$. They were highly resistant to tobramycin $(80.39 \%)$, ciprofloxacin $(79.08 \%)$ and ceftazidime (72.55\%). 16 out of 153 Pseudomonas isolates were resistant to imipenem, among which 12 were MBL producers.

Enterobacteriaceae were least resistant to imipenem $(3.08 \%)$, tigecycline (7.05\%), polymyxin B $(9.69 \%)$ and levofloxacin (14.01\%). Resistant rate for Ampicillin-sulbactam, Piperacillin tazobactam and amikacin was $52.42 \%, 49.33 \%$ and $41.4 \%$ respectively. They showed highest resistance to cephalosporins ( $82 \%$ to $98 \%)$, cotrimoxazole $(81.93 \%)$ tetracycline $(78.41 \%)$, gentamycin (77.97\%) and ciprofloxacin (71.37\%). $72.25 \%, 19.6 \% \& 3.08 \%$ of total Enterobacteriaceae were ESBL, AmpC and Carbapenamase producers respectively.

The most effective antibiotics for Acinetobacter spp which showed least resistance were polymyxin B $(2.47 \%)$, imipenem $(7.4 \%)$, tigecycline $(9.88 \%)$, levofloxacin (17.28\%), Ampicillin-sulbactam $(19.75 \%)$ and Piperacillin - tazobactam $(20.98 \%)$. Resistance to tetracycline, gentamycin and cotrimoxazole amounts $75 \%$ to $84 \%$. Acinetobacter spp showed considerable resistant to cephalosporins (80-95\%), 6 out of 81 (7.4\%) Acinetobacter isolates were MBL producers.

$59.46 \%$ of $S$. aureus isolated were MRSA. All S. aureus isolates were sensitive to vancomycin, tigecycline and linezolid. S. aureus showed good sensitivity to levofloxacin $(78.38 \%)$ and chloramphenicol $(64.86 \%)$.

\section{Discussion}

It is well documented that the higher rates of infection and mortality among ICU patients are mostly related to factors such as exposure to invasive procedures, underlying disease conditions, duration of stay in the ICU, infection sites and association with nosocomial multidrug-resistant pathogens [6]. Our study included both major infection sites and types of organisms and their resistance to commonly used antibacterial agents. Percentage of microbial isolation is variable in different studies. In this study infection rate among ICU patients is $48.38 \%$ which is comparable to $51 \%$ in previous study; International Study of the Prevalence and Outcomes of Infection in Intensive Care Units [1]. Infection rate among ICU patients due to gram-negative bacteria is significantly higher than gram-positive bacteria and candida isolates. There is quite difference in mode of infection sites in Medical/ Pediatric ICU and surgical ICU. Urinary tract infections and Skin and soft tissue infections were most common infection in medical/ pediatric ICU and surgical ICU respectively.

Rate of infection was higher in lower (0-9 years) because they are more susceptible to infection due to various reasons along with their weak immune system and higher (>60years) age group which can be correlate with debilitating condition, immune status of the patients and aging in this age group. In the present study, most common bacterial pathogen in ICU infections was Pseudomonas which is in accordance with the results of several similar studies conducted worldwide [1, 7-9]. The other common organisms involved in ICU infection were Klebsiella, E. coli, Acinetobacter and Staphylococcus aureus. In Asian countries the most frequent pathogen isolated from infection in ICU are Pseudomonas, E. coli,
Klebsiella and staphylococcus [7]. In our study, E. coli was the most frequent pathogen obtained from patients with urinary tract infection. This is similar to previous studies in terms of how frequent this pathogen was detected [10-13].

Antibiotic use contributes to the emergence of antimicrobial resistance in gram positive as well as gram negative bacteria. In developing countries, antibiotics are prescribed for $44 \%-97 \%$ of patients in hospital, often inappropriately [7, 14-15]. Overall 59.46\% of all Staphylococcus aureus associated infections in ICU were caused by methicillin-resistant strains. Total $72.25 \%, 19.6 \%$ \& $3.08 \%$ of Enterobacteriaceae were ESBL, AmpC and Carbapenamase producers respectively.

Imipenem and Polymyxin B was the most sensitive antibiotic against gram negative isolates which should be reserved for multi resistant isolates while in case of $S$. aureus, vancomycin, linezolid and tigecycline were most sensitive antibiotic. Piperacillin - tazobactam, levofloxacin, aztreonam and amikacin were effective antibiotics against $P$ seudomonas isolates for which sensitivity ranges was $65 \%$ to $83 \%$. Similarly other gram negative isolates including Enterobacteriaceae and Acinetobacter spp. showed good sensitivity to levofloxacin, ampicillin-sulbactam and piperacillin-tazobactam. Most of the gram negative isolates were resistant to all betalactams, gentamycin, tobramycin, tetracycline and cotrimoxazole.

\section{Conclusion}

Role of microbiologist in prevention of Hospital Acquired Infection has proven vital due to emergence of new pathogens and transformation into resistances to variety of anti-microbial agents. Many lives are either saved or lost in the ICU of every major hospital because of pitfalls in accurate diagnosis or in lack of proper monitoring. Infection is a major cause of adverse patient outcome. Close interactions between microbiologists and intensive's can save many more lives. The choice of antibiotics in the ICU patients is usually empiric. Therefore, the ongoing surveillance of antibiotic susceptibility patterns of predominant bacteria is a primary effort to guide the clinician in choosing empirical or directed therapy appropriately, especially in ICU setting. In this study we showed that antimicrobial resistance pattern of most common pathogens in ICU may cost lives of the patients as well as it is indirect costing to the hospital by way of acquired infection. We hope that this data will be useful for healthcare professionals in deciding antibiotic cycling policies and the analysing emergence of new pathogens in critical areas like ICU.

\section{References}

[1] Jean-Louis Vincent, Jordi Rello, John Marshall (2009) JAMA, 302(21), 2323-2329.

[2] Patwardhan R.B., Dhakephalkar P.K., Niphadkar K.B. and Chopade B.A. (2008) Indian J. Med. Res., 128, 178-187.

[3] Karin A. Thursky, Kirsty L. Buising, Narin Bak, Lachlan Macgregor, Alan C. Street, C. Raina Macintyre, Jeffrey J. Presneill, John F. Cade And Graham V. Brown (2006) International Journal for Quality in Health Care, 18(3), 224-231.

[4] Carmen Lorente, Yolanda del Castillo, and Jordi Rello (2002) Current Opinion in Critical Care, 8, 461-464. 
Raval P.N., Patel P.G., Patel B.V., Soni S.T., Bhatt S.K., Vegad M.M., Vaghela G.M., Yadav K.S., Shirish Patil and Goswami H.M.

[5] Clinical and Laboratory Standard Institute. Performance standards for antimicrobial susceptibility.

[6] Asem A. Shehabi and Izdiad Baadran (1996) Eastern Mediterranean Health Journal, 2(3), 515-520.

[7] Maksum Radji, Siti Fauziah, Nurgani Aribinuko (2011) Asian Pacific Journal of Tropical Biomedicine, 39-42.

[8] Mahin Jamshidi, Sedigheh Javadpour, Tasnim Eghbal Eftekhari, Nahid Moradi and Faegheh Jomehpour (2009) African Journal of Microbiology Research, 3(10) 590-594.

[9] Tirthankar Mukherjee, Pramod K., Gita Srinivasan, Medha Y. Rao (2005) Journal of the Indian Academy of Geriatrics, 1(2).

[10]Nwadioha S.I., Nwokedi E.E., et al, The Internet Journal of Infectious Diseases, ISSN: 1528-8366.

[11]Jombo G.T.A., Ayilara A.O., Bello N.K., Dakum, Enenebeaku M.N.O. (2005) Journal of Medical laboratory Sciences, 14(2), 54-60.

[12] Kaul S., Brahmadathan K.N., et al (2007) IJMM, 25(3), 203205.

[13]Abdulrahman Abdulla Kader, Kumar A., et al (2004) Saudi J. Kidney Dis. Transplant, 15(2), 135-139.

[14]Hu S., Liu X., Peng Y. (2004) J. Infect., 48, 117-118.

[15]Ansari F. (2001) Eur. J. Clin. Pharmacol., 57, 547-551. 